\title{
A View of Adenomatoid Odontogenic Tumor in Ameloblastoma: A Hybrid Variant
}

\author{
Sapna Chandran Lathakumari ${ }^{1}{ }^{\infty}$, Priya Thomas $^{2}$
}

\begin{abstract}
Aim: Odontogenic tumors (OTs) are a group of lesions encountered in the jaw with diverse histologic patterns. Few cases of hybrid OT have been reported in the literature, and the current knowledge in this entity is limited principally due to its sporadic occurrence and diverse complex histologic picture.

Background: Few cases of hybrid presentation of other OT with ameloblastoma are reported previously. This is most often a dilemma to the clinicians and pathologists. This may delay the proper treatment strategy in patients, leading to comorbidities. Ameloblastoma is a benign OT characterized by local aggressiveness, unlimited growth potential, malignant transformation, and metastasis.

Case description: This paper reports a case of a hybrid variant of ameloblastoma with adenomatoid odontogenic tumor (AOT) seen in the maxillary region with calcifications and a history of three times recurrence. AOT is believed to be hamartomatous in origin derived from odontogenic epithelium of dental lamina or its remnants. To date, very few cases of this entity have been reported, and it is considered to be either true hybrids or an anomalous histodifferentiation or morphodifferentiation in origin. It is demonstrated with combination of either typical or atypical AOT with ameloblastoma. This type of lesion needs adequate treatment and follow-up modalities.

Conclusion: Hybrid entities are very rare tumors comprising two different tumors each of which conforms to an exactly defined tumor category. Therefore, the identification and treatment of such an entity are extremely important.

Clinical significance: Cases with long-term history and recurrences should be evaluated with proper clinical and histological examination.

Keywords: Adenomatoid odontogenic tumor, Ameloblastoma, Hybrid, Odontogenic tumors, Recurrence.

Journal of Health Sciences \& Research (2021): 10.5005/jp-journals-10042-1100
\end{abstract}

\section{BACKGROUND}

Odontogenic tumors (OTs) originating from odontogenic epithelium have the potential for diverse differentiation and mesenchymal induction. ${ }^{1}$

Ameloblastoma is a benign, locally destructive, and invasive tumor ranking second among the common OTs. ${ }^{2}$ It is observed mostly in the posterior aspect of jaws and has a high rate of recurrence despite adequate surgical removal. The revised classification of the World Health Organization (WHO) (2017) has modified the histologic categorization of ameloblastoma. ${ }^{3}$

Adenomatoid odontogenic tumor (AOT) is an uncommon, benign OT of the jaw, occurring mostly in association with impacted maxillary cuspid. AOT accounts for 2.2 to $7.1 \%$ of all OTs and is ranked fourth or fifth in the order of occurrence. It was proposed by Steensland in 1905 and later described by Philipsen and Birn in 1907. ${ }^{4}$ Some consider it as a hamartomatous entity that originates from reduced enamel epithelium; but considering its benign, nonneoplastic, and noninvasive nature with slow but progressive growth, it is treated in a conservative manner. ${ }^{5}$

According to the latest classification, 2017, both these lesions are classified under "Benign epithelial odontogenic tumors."

\section{Case Description}

A 51-year-old male patient reported to the outpatient department of Annoor Dental College, Ernakulam, on November 1, 2016. The patient complained of swelling in the right side of the face for 6 months with progressive enlargement together with slight pain and discomfort. He gives a history of surgery undertaken twice at the same region, initially due to an impacted tooth and later
${ }^{1}$ Department of Oral Pathology and Microbiology, Mar Baselios Dental, Ernakulam, Kerala, India

${ }^{2}$ Department of Oral Pathology and Microbiology, Annoor Dental College and Hospital, Ernakulam, Kerala, India

Corresponding Author: Sapna Chandran Lathakumari, Department of Oral Pathology and Microbiology, Mar Baselios Dental, Ernakulam, Kerala, India, Phone: +91 9496749846, e-mail: sapnanairs@gmail.com

How to cite this article: Lathakumari SC, Thomas P. A View of Adenomatoid Odontogenic Tumor in Ameloblastoma: A Hybrid Variant. J Health Sci Res 2021;12(1):21-25.

Source of support: Nil

Conflict of interest: None

after 15 years due to recurrent swelling. There was no relevant medical or family history nor any adverse habit association. On inspection, an oval-shaped diffuse swelling of size $3 \times 2 \mathrm{~cm}$ was observed. The overlying mucosa displayed normal color and texture extending from right central incisor to first molar mesiodistally and superoinferiorly from free marginal gingiva to buccal vestibule causing its obliteration. There is a single unit bridge extending from the right central incisor to the first premolar. On palpation, the swelling was firm in consistency, slightly tender with an egg-shell crackling of buccal cortical bone. Orthopantomogram (OPG) and computed tomography (CT) scan of the face was advised for the patient. Intraoral periapical (IOPA) (Fig. 1) and OPG revealed radiopacity coronally involving pulp canal showing crown placed and mixed radiopacity and radiolucency apically (Fig. 2). CT showed a cystic lesion involving the maxillary sinus (Fig. 3). According to clinical and radiological findings, a provisional 


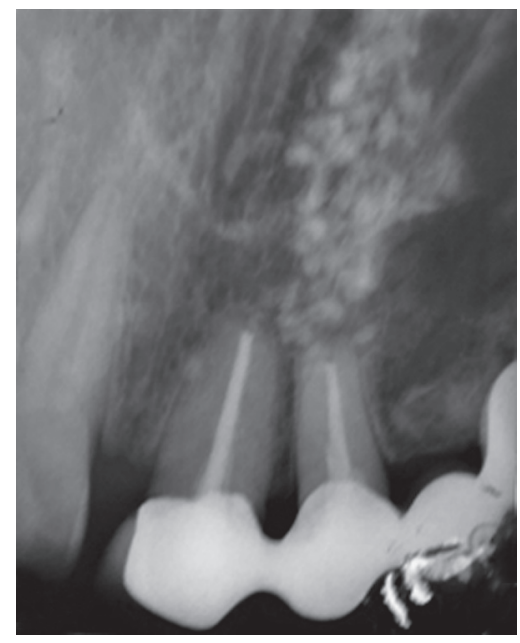

Fig. 1: IOPA showing mixed radiopacity and radiolucency noticed in the apical area 11,12

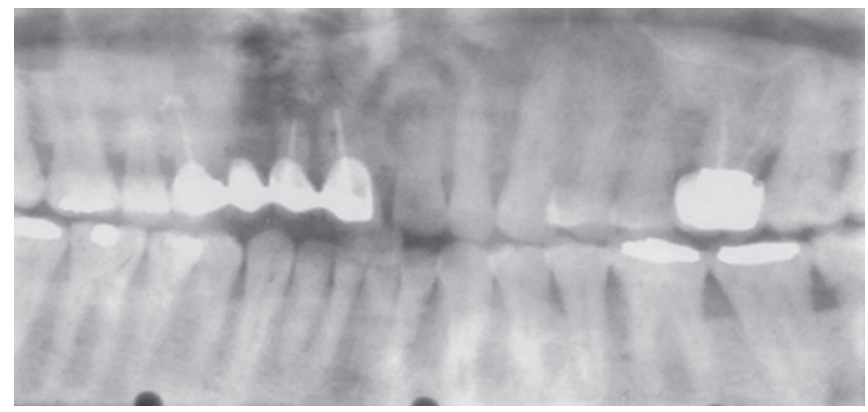

Fig. 2: OPG showing mixed radiopacity and radiolucency extending to 13

diagnosis of the radicular cyst was considered. Differential diagnosis included unicystic ameloblastoma (UA), calcifying odontogenic cyst, calcifying epithelial odontogenic tumor (CEOT), and AOT. Aspiration using 18 gauge was performed and showed a negative result. The lesion was then completely excised under local anesthesia with adequate margin. The adjacent bone was well curettaged due to a history of recurrence. The surgical specimen was then sent for biopsy for further evaluation.

Macroscopically two tissues with firm consistency and irregular borders, brown to white in color measuring $1.5 \times 1 \times 0.5 \mathrm{~cm}$, were received and processed (Fig. 4).

The histopathological examination revealed an OT consisting of both follicular and plexiform ameloblastomatous areas with dispersed multiple and smaller foci of AOT pattern. There was evidence of proliferating odontogenic epithelium arranged in interconnecting cords and sheets (Fig. 5). Few cells were found to be cuboidal with a round to ovoid, vesicular, and hyperchromatic nuclei. Tumor cells were arranged in rosettes, ducts, and involuted patterns with hyaline droplets and mucin pools. In between the nodules, ducts, and rosettes, spindle- and stellate-shaped cells were present in the form of sheets. Few odontogenic epithelial islands with central areas of stellate reticulum were noted. Both the ameloblast-like cells and duct-like structures manifested tall columnar epithelial cells. The connective tissue stroma was scanty with vascular channels and calcifications present in isolated areas (Fig. 6). There was evidence of induction materials and cystic

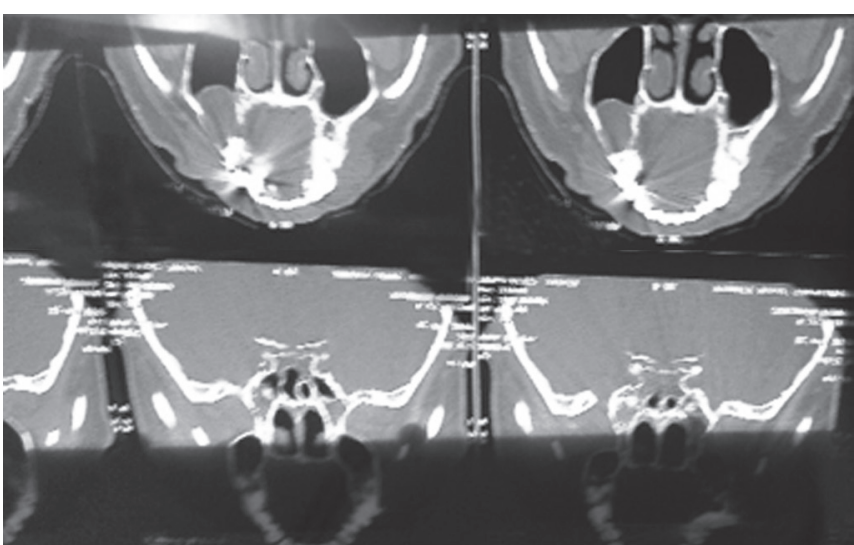

Fig. 3: CT showing a cystic lesion

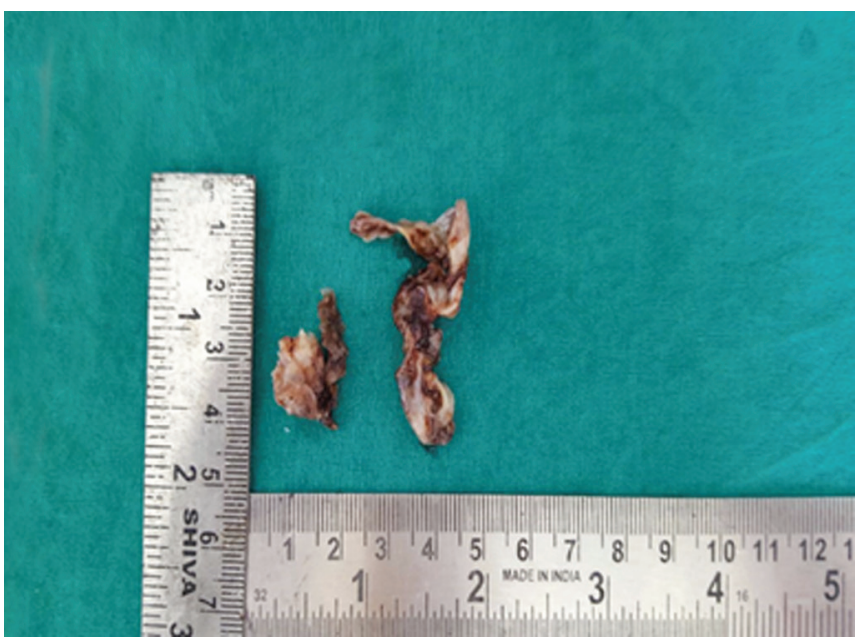

Fig. 4: Macroscopic appearance of the specimen

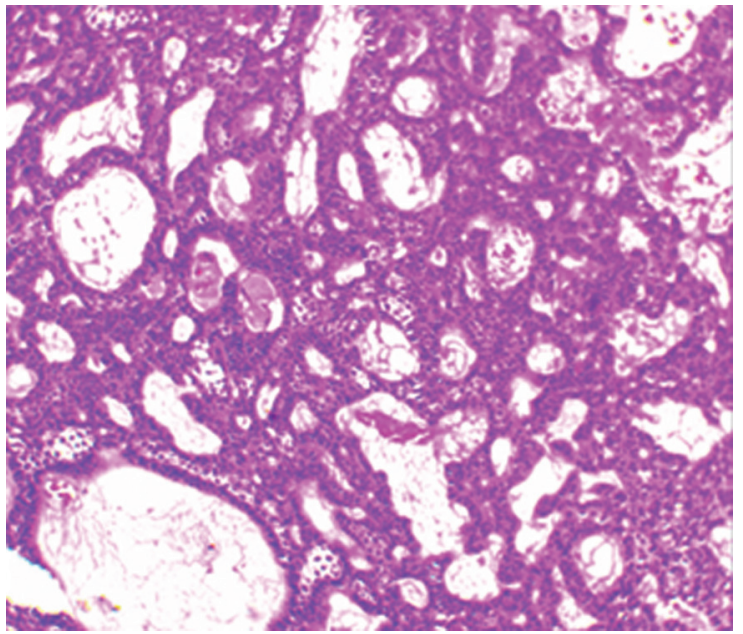

Fig. 5: Proliferating odontogenic islands in interconnecting cords and sheets (H\&E,100X)

changes in the atypical arrangements, which resembled the appearance of AOT; the classical eosinophilic materials, which are typically seen in AOT, were not appreciated. These features were suggestive of ameloblastoma with features of AOT (Fig. 7). 


\section{Discussion}

Hybrid tumors are composed of two or more different tumoral entities in a single neoplasm that arises within a definite topographical region. Hybrid OT types have been documented, but their occurrences are not very common. The present case is unique as excisional biopsy showed one area of AOT features with predominating plexiform ameloblastomatous entity. This type of hybrid variant with a solid component of ameloblastoma was first reported in $2014 .{ }^{6}$ In the literature, various studies have reported infrequent clinicopathological findings in ameloblastomas. Two cases of AOT with UA were reported by Raubenheimer et al. study in 1995, where he observed variations in 108 ameloblastoma cases. ${ }^{7}$

A similar case was reported by Jivan et al., showing the existence of extrafollicular AOT and UA. ${ }^{8}$

Few other cases with hybrid tumors like AOT with CEOT, AOT arising from calcifying odontogenic cyst, and AOT developing in ameloblastoma have also been reported in the literature.

AOT and ameloblastoma are odontogenic epithelial tumors categorized under the same classification. They are usually found in association with impacted tooth seen around second decade. This hybridization of AOT with ameloblastoma was designated "adenoid" ameloblastoma (AA). This attribution was mainly meant for specifying those tumors that expose impressive occurrence of AOT-like areas along with the tumoral ameloblastic component. This makes the diagnosis of such an entity extremely challenging. ${ }^{9,10}$ Previously few cases of AOT association with UA were reported. ${ }^{10}$ Few other tumors associated with AOT are CEOT, and AOT arising from the calcifying odontogenic cyst.

OTs develop from odontogenic epithelium, which is capable for diverse differentiation and mesenchymal induction. Some studies, with the few exceptions, suggest these ambiguous tumors should not generally be considered true hybrids; rather they should be identified simply as anomalous histodifferentiation and/or morphodifferentiation process. ${ }^{10,11}$

Ameloblastoma is a slow-growing, locally destructive, and invasive tumor with a high rate of recurrence. It is the second most common OT accounting for about $1 \%$ of all oral tumors and about $18 \%$ of OTs. It is diagnosed mainly in the third to fifth decades of life, with almost equal sex predilection. ${ }^{12}$

Earlier classification of OTs of the WHO (2005) had classified the variants of ameloblastomas into solid or multicystic, the extraosseous ameloblastoma, the desmoplastic, and the unicystic type. $^{13}$

According to the latest WHO (2017) classification, ameloblastoma is mainly divided into:

- Conventional ameloblastoma,

- Unicystic type ameloblastoma,

- Extraosseous/peripheral type, and

- Metastasizing ameloblastoma.

The earlier classification was considered highly complex, and hence, the latest classification discarded the solid or multicystic component, since this pattern mostly refers to the typical conventional pattern and the term "cystic," creating confusion with the "unicystic" counterpart. Similarly, desmoplastic ameloblastoma also lost its specificity and was categorized as one of the histologic variants of conventional ameloblastoma. This entity also like other variants had a distinct histological pattern but in a diagnostic view had no evidence of any differences in behavior. But peripheral and UA showed a distinct behavior and clinicopathological

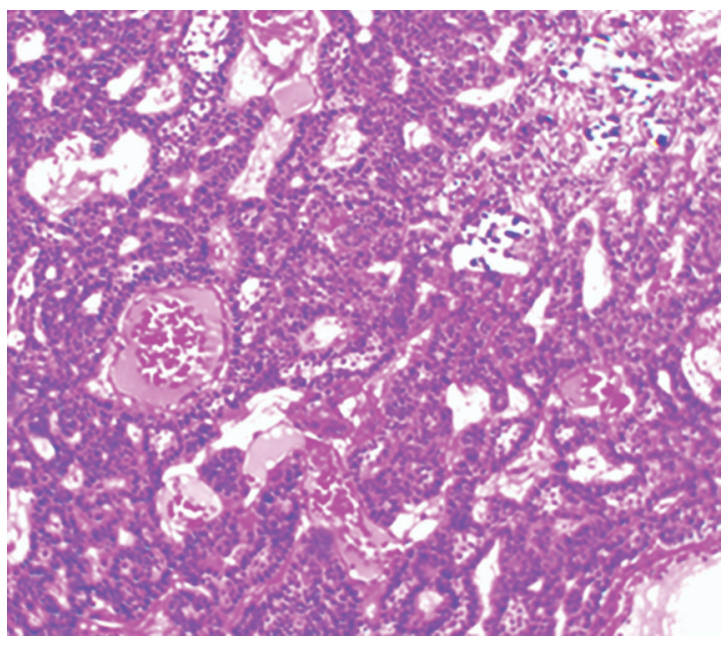

Fig. 6: Few calcifications present (H\&E, 100X)

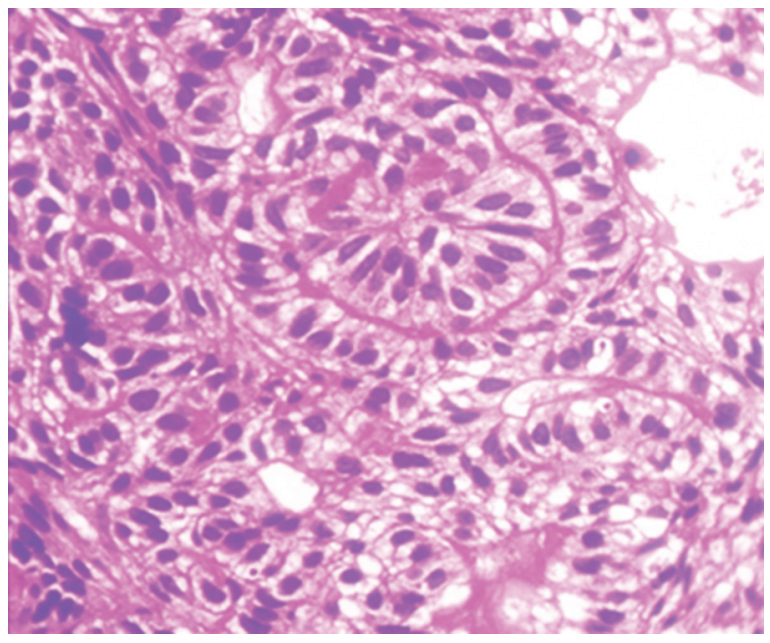

Fig. 7: Tumor cells showing ductal pattern but having reversal pattern (H\&E, 400X)

characteristics; therefore, they are retained as a specific subtype. The subdivisions of UA are maintained as previously. Metastasizing ameloblastoma was reshuffled from the section of ameloblastic carcinoma to a type of benign conventional ameloblastoma, defining it as a histologically benign typical ameloblastoma that metastasizes to distant sites. ${ }^{3}$

Ameloblastoma derived from the remnants of Rathke's pouch or a misplaced enamel organ is known as craniopharyngiomas. In few cases, malignant transformation of ameloblastomas has been reported, resulting in the formation of ameloblastic carcinomas and malignant ameloblastomas depending on cytological dysplasia and metastasis, respectively. ${ }^{14,15} \mathrm{~A}$ rare variant of ameloblastic carcinoma with spindle cell expression showing extensive metastasis and poor outcome has been reported in the previous literature. ${ }^{16}$

AOT is also referred to as " $2 / 3$ rd tumor" as $2 / 3$ rd of cases occur in the maxilla, $2 / 3$ rd occur in young females, $2 / 3$ rd are associated with unerupted teeth, and $2 / 3 \mathrm{rd}$ are associated with impacted canine and lateral incisor. It accounts for about 1 to $9 \%$ of all OTs. 
It was first proposed by Stafne in $1948 .^{17}$ The most widely accepted abbreviated terminology for adenomatoid odontogenic tumor as AOT was proposed by Philipsen and Birn. ${ }^{4}$

This entity was earlier considered as a variant of ameloblastoma $^{18}$, so it was also known as pseudo adenomatous ameloblastoma, odontoameloblastic tumor, cystic complex composite odontoma, etc. ${ }^{19}$ Various terminologies were proposed like adenoameloblastoma, ameloblastic adenomatoid tumor, epithelioma adamantinoma, or teratomatous odontoma. ${ }^{20}$

There are three variants of AOT-follicular, extrafollicular, and peripheral. Follicular variant is associated with the unerupted tooth, while extrafollicular has no relation to the impacted tooth. Follicular type is the most common growth type. The peripheral variant is also observed, which appears in the gingiva. ${ }^{21}$

In certain areas, nodular islands forming duct-like structures are observed, which at higher magnification reveal tall columnar epithelium. ${ }^{22}$ Variable amounts of calcified materials are also reported in most AOTs suggestive of inductive changes in connective tissue. ${ }^{23}$

AOT should be radiologically differentiated from the dentigerous cyst. AOT usually shows radiolucency usually surrounding the coronal and radicular aspect of the involved tooth, whereas dentigerous cyst involves only the coronal aspect of the impacted tooth. ${ }^{24}$ AOT also expresses numerously dispersed or clustered radiopaque foci showing a thin radiopaque line and discrete radiopaque foci. Therefore, radiolucency with numerous radiopaque foci (particularly when the radiolucency surrounds a portion of the root or entire tooth) gives differentials like AOT, calcifying cystic OT. ${ }^{25}$

Few cases of aggressive behavior of AOT where an intracranial extension from the maxilla and cases of recurrence have been reported. ${ }^{26,27}$

The present case showed a combination of ameloblastoma intermingled with features of AOT and few areas of calcification. This case also emphasizes the chances of recurrence. Few such combinations of ameloblastoma with AOT, including dentinoid features and showing recurrence, have been reported (AA with dentinoid). ${ }^{28,29}$

\section{CONCLUSION}

It is thus important to report rare cases of such hybrid tumors as treatment for each entity differs. AOT requires only surgical excision. But when it is present in association with UA or hybrid tumors, treatment may be radical excision and long-term follow-up may be required. Prognosis will be good if complete excision is done with close follow-up. ${ }^{30}$ We have done follow-up for 2 years, and the lesion has healed uneventfully.

\section{Clinical Significance}

Hybrid entities often result in controversies among the clinicians and pathologists. But identification and treatment planning of such entities are of utmost importance to prevent recurrences and other comorbidities.

\section{ACKNOWLEDGMENTS}

I would like to express my gratitude to all those who provided me the possibility to complete this case report.

\section{OrCID}

Sapna Chandran Lathakumari 아 https://orcid.org/0000-0002-30741606

\section{References}

1. Gorlin RJ, Pindborg JJ, Clausen FP, et al. The calcifying odontogenic cyst: a possible analogue of the cutaneous calcifying epithelioma of malherbe. Oral Surg Oral Med Oral Pathol 1962;15:1235-1243. DOI: 10.1016/0030-4220(62)90159-7.

2. Dhanuthai K, Chantarangsu S, RojanawatsirivejS, et al. Ameloblastoma: a multicentric study. Oral Surg Oral Med Oral Pathol Oral Radiol 2012;113(6):782-788. DOI: 10.1016/j.00oo.2012.01.011.

3. Speight PM, Takata T. New tumour entities in the 4th edition of the World Health Organization Classification of Head and Neck tumours: odontogenic and maxillofacial bone tumours. Virchows Archiv 2018:472(3):331-339. DOI: 10.1007/s00428-017-2182-3.

4. Philipsen HP, Birn H. The adenomatoid odontogenic tumour. Ameloblastic adenomatoid tumour or adenoameloblastoma. Acta Pathol Microbiol Scand 1969;75(3):375-398.

5. Nath S, Shahi AK, Pulikkotil SJ, et al. Hybrid variant of adenomatoid odentogenic tumour. Biomed J Sci Tech Res 2017;1(3):682-685. DOI: 10.26717/BJSTR.2017.01.000273.

6. Yamazaki M, Maruyama S, Abé T, et al. Hybrid ameloblastoma and adenomatoid odontogenic tumor: report of a case and review of hybrid variations in the literature. Oral Surg Oral Med Oral Pathol Oral Radiol 2014;118(1):e12-e18. DOI: 10.1016/j.oooo.2013.08.032.

7. Raubenheimer EJ, Heerden WF, Noffke CE. Infrequent clinicopathological findings in 108 ameloblastomas. J Oral Pathol Med 1995;24(5):227-232. DOI: 10.1111/j.1600-0714.1995.tb01172.x.

8. Jivan V, Altini M, Meer S, et al. Adenomatoid Odontogenic Tumour (AOT) originating in a unicystic ameloblastoma: a case report. Head Neck Pathol 2007;1(2):146-149. DOI: 10.1007/s12105-007-0005-6.

9. Loyola AM, Cardoso SV, de Faria PR, et al. Adenoid ameloblastoma: clinicopathologic description of five cases and systematic review of the current knowledge. Oral Surg Oral Med Oral Pathol Oral Radiol 2015;120(3):368-377. DOI: 10.1016/j.000o.2015.05.011.

10. Ide F, Horie N, Shimoyama T, et al. So-called hybrid odontogenic tumors: do they really exist? Oral Med Pathol 2001;6(1):13-21. DOI: 10.3353/omp.6.13.

11. Thesleff I, Vaahtokari A, Ketunen P, et al. Epithelial-mesenchymal signaling during tooth development. Connect Tissue Res 1995;32 (1-4):9-15. DOI: 10.3109/03008209509013700.

12. Reichart PA, Philipsen HP, Sonner S. Ameloblastoma: biological profle of 3677 cases. Eur J Cancer B Oral Oncol 1995;31B:86-99. DOI: 10.1016/0964-1955(94)00037-5.

13. Ledesma-Montes C, Mosqueda-Taylor A, Carlos-Bregni R, et al. Ameloblastomas: a regional Latin-American multicentric study. Oral Dis 2007;13(3):303-307. DOI: 10.1111/j.1601-0825.2006.01284.x.

14. Lee SK, Kim YS. Current concepts and occurrence of epithelial odontogenic tumors: I. Ameloblastoma and adenomatoid odontogenic tumor. Korean J Pathol 2013;47(3):191. DOI: 10.4132/ KoreanJPathol.2013.47.3.191.

15. Paulus W, Stöckel C, Krauss J, et al. Odontogenic classification of craniopharyngiomas: a clinicopathological study of 54 cases. Histopathology 1997;30(2):172-176. DOI: 10.1046/j.1365-2559.1997. d01-584.x.

16. Kawauchi S, Hayatsu Y, Takahashi M, et al. Spindle-cell ameloblastic carcinoma: a case report with immunohistochemical, ultrastructural, and comparative genomic hybridization analyses. Oncol Rep 2003;10(1):31-34. DOI: 10.3892/or.10.1.31.

17. Lee JK, Lee KB, Hwang BN. Adenomatoid odontogenic tumor: a case report. J Oral Maxillofac Surg 2000;58(10):1161-1164. DOI: 10.1053/ joms.2000.9581.

18. Ghosh LS. Adamantinoma of the upper jaw: report of a case. Am J Pathol 1934;10(6):773. 
19. Philipsen HP, Reichart PA, Zhang KH, et al. Adenomatoid odontogenic tumor: biologic profile based on 499 cases. J Oral Pathol Med 1991;20(4):149-158. DOI: 10.1111/j.1600-0714.1991.tb00912.x.

20. Becker T, Buchner A, Kaffe I. Critical evaluation of the radiological and clinical features of adenomatoid odontogenic tumour. Dentomaxillofac Radiol 2012;41(7):533-540. DOI: 10.1259/dmfr/19253953.

21. Philipsen HP, Reichart PA. Adenomatoid odontogenic tumour: facts and figures. Oral Oncol 1999;35(2):125-131. DOI: 10.1016/s13688375(98)00111-0.

22. Sharma N, Passi S, Kumar VV. Adenomatoid odontogenic tumor: as an unusual mandibular manifestation. Contemp Clin Dent 2012;3(Suppl. 1):S29-S32. DOI: 10.4103/0976-237X.95100.

23. de Matos FR, Nonaka CF, Pinto LP, et al. Adenomatoid odontogenic tumor: retrospective study of 15 cases with emphasis on histopathologic features. Head Neck Pathol 2012;6(4):430-437. DOI: 10.1007/s12105-012-0388-x.

24. Nomura M, Tanimoto K, Takata T, et al. Mandibular adenomatoid odontogenic tumor with unusual clinicopathologic features. J Oral Maxillofac Surg 1992;50(3):282-285.

25. Chindasombatjaroen J, Poomsawat S, Kakimoto N, et al. Calcifying cystic odontogenic tumor and adenomatoid odontogenic tumor: radiographic evaluation. Oral Surg Oral Med Oral Pathol Oral Radiol 2012;114(6):796-803. DOI: 10.1016/j.00oo.2012.08.452.

26. Sandhu SV, Narang RS, Jawanda M, et al. Adenomatoid odontogenic tumor associated with Dentigerous cyst of the maxillary antrum: a rare entity. J Oral Maxillofac Pathol 2010;14(1):24-28. DOI: 10.4103/0973-029X.64308.

27. Toida M, Hyodo I, Okuda T, et al. Adenomatoid odontogenic tumor: Report of two cases and survey of 126 cases in Japan. J Oral Maxillofac Surg 1990;48(4):404-408. DOI: 10.1016/02782391(90)90441-4.

28. Handschel JG, Depprich RA, Zimmermann AC, et al. Adenomatoid odontogenic tumor of the mandible: review of the literature and report of a rare case. Head Face Med 2005;1(1):3. DOI: 10.1186/1746160X-1-3.

29. Evans BL, Carr RF, Phillipe LJ. Adenoid ameloblastoma with dentinoid: a case report. Oral Surg Oral Med Oral Pathol Oral Radiol Endodontol 2004;98(5):583-588. DOI: 10.1016/j.tripleo.2004.02.077.

30. Sathyanarayana VK, Srigiri $H$, Cheemalavagupalli $M$, et al. A rare case of adenomatoid odontogenic tumour with unicystic ameloblastoma. J Clin Diagn Res 2017;11(2):ZJ05. DOI: 10.7860/ JCDR/2017/23623.9412. 